\title{
User Experience in Cross-channel Ecosystems
}

\author{
David Benyon \\ Centre for interaction Design \\ Edinburgh Napier University \\ Edinburgh EH10 5DT, UK \\ d.benyon@napier.ac.uk
}

\author{
Andrea Resmini \\ Jönköping International Business School \\ Jönköping, \\ Sweden \\ Andrea.resmini@ju.se
}

\begin{abstract}
Recent developments in information and communication technologies have left interaction design and human-computer interaction $(\mathrm{HCl})$ with something of a conceptual gap. The distinction between physical and digital spaces is increasingly blurred. Cloud-based services have enabled a separation of information content from device so that content can be accessed and manipulated across multiple devices and locations. The user experience (UX) frequently needs to deal with activities that transition across physical and digital spaces and ecosystems of devices and services. Designers can no longer prescribe the journey or curate experiences simply as isolated interactions. Instead, UX must be consistently spread across touchpoints, channels, and device ecosystems. Our contribution to the development of UX, interaction design, and information architecture is to appeal to the notions of cross channel user experiences and blended spaces. Information architecture is the pervasive layer that underlies interactions that cross services, devices and blended physical and digital spaces. Information architecture is the structure within which the UX unfolds. From this perspective, we highlight the importance of creating meaningful places for experience and navigation through blended spaces.
\end{abstract}

Blended space, contextual blending, cross-channel, place, place-making, user experience, information architecture.

\section{INTRODUCTION}

Recent developments in information and communication technologies have left interaction design and human-computer interaction $(\mathrm{HCl})$ with something of a conceptual gap. As Mitchell (2004) notes

"(o)nce there was a time and a place for everything; today, things are increasingly smeared across multiple sites and moments in complex and often indeterminate ways". (p. 14)

The distinction between physical and digital spaces is becoming increasingly blurred, not only through the deployment of augmented reality or virtual reality interfaces, but also through the flow of content and information made possible by personal and portable devices. Many different products and services bridge over physical and digital space, from Google Maps to Yelp to Uber. Mobile computing, sensors and actuators embedded in the physical world and location-based services triggered by GPS or Bluetooth beacons make interaction conditional on context and physical location. The user experience is spread across multiple subjects, devices, and spaces, yet most of the interaction design literature still focuses on single location interactions.

In this paper, we address this conceptual gap and identify the different roles information architecture and UX design play when experiences unfold over time and across digital and physical spaces.

We provide a brief review of recent design literature before introducing two key ideas that address the conceptual gap: blended spaces, spaces where some physical and digital spaces have been designed to work together to provide a particular UX; and cross-channel ecosystems, constructs that Resmini and Lacerda (2016) define as

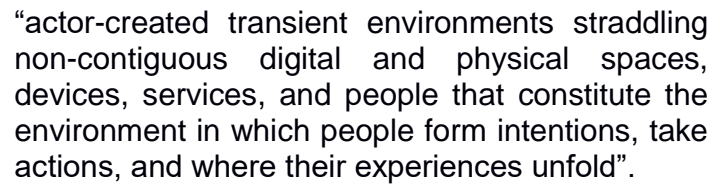
non-contiguous digital and physical spaces, devices, services, and people that constitute the environment in which people form intentions, take actions, and where their experiences unfold".

Bringing these ideas together enables us to introduce a more systemic perspective on interaction 
design that is closer to architecture than product design, as we shift the focus away from the individual interactions to a sustained UX. This allows us to approach the many different interfaces, legacy systems, the constraints and opportunities afforded by physical locations, and the spatial and temporal variants strategically rather than tactically. We focus on how people consume and co-create information content on the move, navigating locations that have different characteristics, and unfolding their user experiences in the context of cross channel ecosystems.

\section{BACKGROUND}

The background to this work is complex as we need to bring contributions from interaction design and information architecture together. These are two fields and communities who have relatively separate conferences and meetings and yet deal with closely related aspects of UX. We see many successful examples of cross-channel interaction design, but we see little theoretical or systematic treatment of the new UX that modern-day device and service ecosystems enable. We see a need for interaction design to change to embrace the lack of control that designers have over the devices and services that users choose. We see a need for information architecture to extend its traditional focus on vocabularies and web sites and to embrace the backstage reforms needed to deliver consistent cross-channel experiences.

\subsection{Interaction Design}

The interaction design literature tends to focus on single device interactions. For example, Forlizzi and Battarbee (2004) state experience "is focused on the interactions between people and products, and the experience that results". Even when looking at product ecologies, Forlizzi's emphasis is on interaction with a product (Forlizzi, 2007). Beaudouin-Lafon's (2000) interaction model searches for "a set of principles that guide the design of an interface". Dalsgård and Halskov (2006) discuss experience-oriented design as it relates to the interaction of people with interactive installations, a focus that is maintained in their more recent work (Dalsgård, Halskov and Wiethoff, 2016; Falck and Halskov, 2013. Jung and Stolterman (2012) explicitly state that their model for interaction design is artefact-focused.

As much as interaction design theory has then so far concerned itself on the individual artefact, traditional definitions of user experience also have been focusing on the single product or service. Shedroff (2010) maintains that user experience is "the overall experience, in general or specifics, a user, customer, or audience member has with a product, service, or event". Hassenzahl (2008) maintains user experience is "a momentary, primarily evaluative feeling (good-bad) while interacting with a product or service". Norman and Nielsen (nd) similarly see it as something that "encompasses all aspects of the end-user's interaction with the company, its services, and its products", ignoring devices and services that are outside the company's control.

Definitions from the practice do not differ: Garrett (2011) says user experience is the experience "the product creates for the people who use it in the real world". Writing for mainstream trade magazine UX Matters, Gabriel-Petit (nd) defines user experience design as "(a) holistic, multidisciplinary approach to the design of user interfaces for digital products, defining their form, behaviour, and content."

This attention to the single artefact, that interaction design shares with product design, runs contrary to the socio-technical changes that are represented by what Jenkins (2006) calls convergence, "the flow of content across multiple media platforms (...) and the migratory behaviour of media audiences".

Media studies offer mature foundations to describe and understand convergence in the current media landscape. According to Jenkins (2008), crossmedia is "a new disruptive pattern of consumption, not of production; the result of the complex interactions of media convergence, participatory culture, and collective intelligence". Crossmedia products are media products expressly designed to be experienced fragmentarily and across a number of different mediums, providing a loose structure in which each part explicitly or implicitly refers to other mediums in the narrative ecosystem.

More recently, transmedia (Jenkins, 2011) acknowledges the role of convergence as a production and co-creation process. Transmedia introduces a systemic approach to experiencing media that considers audience agency and the interlacing of story threads across mediums and in the spaces in between them (lacobacci, 2008).

Crossmedia and transmedia specifically address media and cultural consumption: they limit their analysis to the products of the entertainment industry and present descriptive frameworks whose primary goal is far removed from the processes of design practice. The idea of convergence and its attention to systemic relationships have been extended to UX design through the concept of crosschannel experiences (Resmini and Lacerda, 2016).

This approach is more architectural and global than "designerly" and localized. It prefigures a world of 
spaces, movement, and context rather than a world of artefacts and decontextualized use. Artefacts do not disappear, of course: they are what is contained within the spaces. Their nature becomes that of nodes in a network and elements in a larger, more complex, UX.

\subsection{Place-making}

A complex experience unfolding across a multitude of contexts and physical and digital locations introduces a concern for the container besides that which is contained. This container can be properly described as a place, a layered construct that aggregates subjective emotions, memories, patterns of behaviour and movement to a certain locale (Tuan, 1977). Place-making then identifies the purposeful, designerly creation of a sense of place that people will inhabit and recognize.

Place-making and navigation as movement through environments have so far been only tangential to the conversations on interaction design. Despite her focus on digital-physical systems, Rogers (2006) does not consider the movement of users through the computationally enabled environments that she describes. Hornecker and Burr (2006) include spatial interaction in their interaction design framework but, despite including an explicit consideration of sense of place, do not consider how people move through that space. Ciolfi $(2004 ; 2013)$ is concerned with "those features of a place that are going to shape users' interactions with technologically enhanced environments", and how we can "(modify) the environment and the activities within it by means of technology".

Few designers indeed explicitly consider movement through environments as a core component of the user experience. Benford et al (2009) describe "hybrid trajectories" in their spectator environments and distinguish between the canonical trajectories produced by the designers and the user trajectories experienced by the spectators. In the practice of UX design, navigation is often reduced to the idea of plotting out what is variously called a user journey or a journey map, a synthetic rendition of a specific experience in linear format, as described through point-to-point interactions.

\subsection{Information architecture}

Rosenfeld and Morville (2006) define information architecture as "the structural design of shared information environments". Originally emerging as a practice and field of study in the context of web design, information architecture deals with the problems of structuring, accessing, finding, and using information and content. As such, it has a stronger concern with spatiality, navigation, and wayfinding.

Information architecture can be traced back to seminal work done in the early 1970 s at the Xerox Palo Alto Research Center (PARC) (Resmini and Rosati, 2013), and Richard Saul Wurman's work throughout the 1970s all the way to the 1990s (Wurman, 1990). Information architecture becomes a mainstream activity and a subject of academic research in the late 1990s with the rise of the World Wide Web. Wurman's initial architectural take, where organizing and structuring information is intended as a close analogue to architectural work in physical spaces (Wurman, 1989; 1990) lost traction as theories and methods providing better matches with the disruptive dynamic environment of the early Web were adopted.

More recently, a "third wave" of Information Architecture has revisited Wurman's architectural perspective, concerning itself primarily with the relationship between embodiment, spatiality, and the pervasiveness of information spaces (Resmini, 2014). Hinton (2015) suggests we use information "to create architectures - environmental structures that shape the experience of place". Lucas et al (2012) maintain that information architecture is the application of the architectural principles which give "a collection of objects, a concept or a system (...) a basis for order, structure, change, or growth" to the "domain of information". Resmini and Rosati (2009) adapt Norberg-Schulz's (1987) concept of existential space and its egocentric topology structured via spatial primitives to digital / physical environments. They position place-making as the core characteristic of a successful UX.

\subsection{A new context for design}

Coming from an activity theory perspective, Bodker and Klokmose (2012) have recently characterized interaction design as dealing with "human beings (...) situated in a web of activities and ecologies of technological artifacts". In his reimagining of $\mathrm{HCl}$, Liam Bannon (2011) takes us back to Terry Winograd's view that

\footnotetext{
"beyond the interface, we operate in an interspace that is inhabited by multiple people, workstations, servers, and other devices in a complex web of interactions (...) Computer systems and software are becoming media for the creation of virtualities: the worlds in which users of the software perceive, act, and respond to experiences." (Winograd, 1997, p. 4).
}

We are now 10 years into "third wave" $\mathrm{HCl}$ (Bodker, 2015) with its emphasis on "experience-based use situations". McCarthy and Wright (2004) in their 
treatment of user experience recognize the importance of a spatio-temporal thread to experience, along with the cognitive, sensorial and emotional. Bill Buxton maintains that one of the most significant issues facing computer users is bridging the physical and digital worlds (2001).

David Kirsh (2000), an early proponent of distributed cognition, sees interaction design as focusing on where the body ends and the environment begins, and how we should

\begin{abstract}
"think of an environment as an activity space originally a physical space but virtual spaces qualify as activity spaces as well - populated with resources, tools and constraints in which an agent operates" (p. 35)
\end{abstract}

Kirsh's later work (Kirsh, 2013) on embodied cognition emphasizes that the tools, objects and other resources that are in the environment affect how we think, what we can think and how we act. Kirsh describes the enactive landscape (the environment) as the structure that an agent cocreates with the world (our emphasis).

The agent brings cognitive and cultural models to an environment, but then changes these and uses them given the agent's knowledge of the environment and what the agent is trying to do. The affordances of an environment change according to the knowledge and interests of the agent and the environment extends the abilities of the agent to act by providing resources and objects, both material and informational.

\subsection{Summary}

Most of Kirsh's examples of embodied, extended and distributed cognition emphasize work, intention and agents acting in a goal-oriented manner. Whilst we recognize this is important, UX cannot be reduced to the act of achieving goals. UX is also concerned with acting in a way that reflects one's values, with the hedonic characteristics of action, with affect and cognition, and with personal or social enrichment (Benyon, 2017).

But in line with his view of embodied and embedded experience, designers need to recognize that most of our day-to-day experiences increasingly occur across a blend of digital and physical spaces that are not necessarily contiguous. UX has to take account of the ecologies, services, enabling objects, tools and resources that characterize the activity and information spaces of our environments.

\section{BLENDED SPACES}

As a response to the blurring of physical and digital space, Benyon (2014, p.79) has proposed the concept of a blended space "where a physical space is deliberately integrated in a close-knit way with a digital space". A blended space is where a digital space is carefully designed to commingle with a physical space, thus creating a new type of space with its own emergent structure. Blended spaces have new properties that emerge from the particular combination of physical and digital, creating a new sense of presence and leading to new ways of interacting and novel user experiences (Benyon, 2012).

The idea of blended spaces has been applied to the domain of digital tourism (Benyon, Mival and O'Keefe, 2013; O'Keefe and Benyon, 2016)], and to the design of meeting rooms (Benyon and Mival, 2012). The concept of blending has also been used for the design of ambient assisted living environments for the elderly (Hoshi and Waterworth, 2009) and for the design of a novel table lamp (Wang, 2016). Blending theory has also been used in the context of blended interaction (Jetter, Reiterer, and Geyer, 2014) where the aim is to bring the experiences of real world interactions to user interface design and in other approaches to $\mathrm{HCl}$ and software engineering (Imaz and Benyon, 2007).

Blended spaces draw on the ideas of blending theory, or conceptual integration (Fauconnier and Turner, 2002; Imaz and Benyon, 2007). The main principle of blending theory is that people come to know things through making projections from two mental spaces in different domains that share a part of their structure with a more generic domain. The projections from the input spaces create new relationships in the blend that did not exist in the original inputs.

An important part of blending theory is its grounding in an embodied cognition perspective (Fauconnier and Turner, 2002) and hence the need to design for interactions in a way that best suits people "at a human scale" as opposed to being dictated to by technology. This is reinforced by a significant contribution to blending theory by Ed Hutchins (2005) arguing for the importance of having a "material anchor" to help people ground their new experiences in something concrete.

Benyon (2012; 2014) develops a view of digital and physical spaces in terms of four characteristics: ontology, topology, volatility, and agency. He argues that for the purpose of creating a good UX these four characteristics constitute the structure of a generic space that both physical and digital spaces share 
(Figure 1). Ontology concerns the objects in the space, topology concerns the spatial relationships between objects, volatility concerns how quickly objects change and agency concerns what people and artificial agents can do in the space.

For example, in a digital tourism experience of a heritage park the ontology consisted of the points of interest, the topology concerned how those points of interest were related in terms of direction and distance, volatility concerned the changing displays at the various points of interest and the agency concerned the activities that people could engage in at the points of interest.

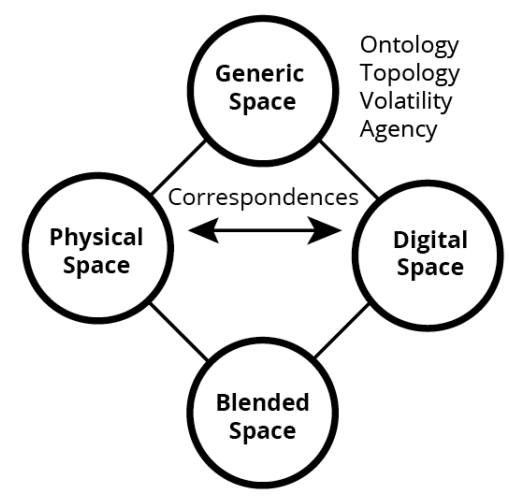

Figure 1: Basic structure of blended spaces

Digital narratives were developed that enabled visitors to explore the park with episodes triggered by a geo-fence around the points of interest and navigation between points provided by a compass (to show direction) and a display to show distance (O'Keefe and Benyon, 2016).

\section{CROSS-CHANNEL ECOSYSTEMS}

Originally a marketing term, cross-channel initially identified a modality of service delivery where a single campaign was driven with a consistent message that was coordinated across mediums such as TV, magazines and in-store displays. It was then introduced to information architecture and UX design (Resmini and Rosati, 2011) as an approach suitable to handle the changes occurring in design practice in response to convergence, the mass penetration of portable devices, the general availability of mobile broadband, and social mediaderived patterns of consumption and co-creation of content.
In this context, the idea of dealing with an ecosystem of connected nodes is put into focus by Resmini and Rosati as they discuss "ubiquitous ecologies" and maintain that the primary element of change is that "information architectures are (now) ecosystems" (Resmini and Rosati, 2009).

As defined more recently by Resmini and Lacerda (2016), cross-channel ecosystems are a superset of the conceptualization of blended spaces offered by Benyon. A cross-channel ecosystem is

"the ecosystem resulting from actor-driven choice,
use, and coupling of touchpoints, either belonging
to the same or to different systems, within the
context of the strategic goals and desired future
states actors intend to explicitly or implicitly
achieve."

A cross-channel ecosystem is a semantic construct structured around the idea of "experiences" that includes people, devices, locations, and software connected through information flows.

\subsection{Cross channel user experiences}

The shift to designing cross-channel experiences implies a certain loss of control and precision in favour of a more strategic view and an acknowledgment that experiences are inevitably user-produced more than user-centred. On one level, this means that organizations and companies such as Wikipedia, Instagram or Uber rely on masses of individuals using their platform to be able to provide these same individuals with a functional service. On another level, the relationship between production and consumption is radically transformed and we cannot simply talk about an "audience" or someone "using a (finished) product" anymore.

This novel agency is the reason for substituting the concept of a "user" with the systems-derived notion of "actor", that of simple "usage" with "co-creation", and for the introduction of a service-dominant logic approach (Vargo and Lusch, 2004). It is in this sense that ecosystems are actor-created constructs. Actors freely move from product to competing product and from service to service unconstrained by company boundaries or physical location. They dynamically shape information-based ecosystems to suit their needs and goals. Such an approach fits complex, pervasive experiences such as "paying one's taxes", "going to the movies", or "commuting to the city centre" which naturally present fuzzy boundaries and which are the ones mostly influenced by personal mobile computing, social interactions, and convergence.

The underlying information architecture that shapes the flow of content and information within the 
ecosystem becomes a primary design element. It offers structural consistency and contextual anchoring to the consumption, co-production, and remediation of information into experiences. It provides the structure that maintains the user experience consistent and coherent.

A cross-channel experience is also an eminently spatially grounded experience. There are strong resonances with Kirsh's (2013) conception, but the spaces that Resmini and Lacerda (2016) consider are much larger than those of Kirsh. Constant consumption and production of a continuous personal stream of content transforms day-to-day activities into complex experiences that flow across physical and digital space and all sorts of everyday environments, from education to healthcare, from traveling to shopping.

\subsection{Key elements of cross-channel ecosystems}

Resmini and Lacerda (2016) establish actors, touchpoints, seams, channels, and the desired future state that actors intend to achieve through any number of tasks or activities as the primary elements of a cross-channel ecosystem. These elements are contextual to the project at hand and polymorphous: for example, a person could be both an actor and / or a touchpoint.

Actors are the subject of the ongoing experience being considered. They could be human actors or software agents. Touchpoints are individual elements within the ecosystem where content is made available to actors. By accessing touchpoints, actors can consume or modify existing content or create more content to be injected into the ecosystem, thus increasing its overall resilience.

A channel identifies a pervasive layer for the interaction with content within the ecosystem. Channels often are high-level, abstract constructs: in an example involving course design in higher education settings, a designer might identify "peer conversations" as a channel where all studentproduced information, in any format, belongs.

It must be noted that channels are a design artefact, whose primary use is to concretely map how information effectively flows through the ecosystem regardless of medium, location, or an individual actor's awareness. They are part of the information architecture, and could reflect the formal sectioning provided by an enterprise architecture model, be the result of content analysis on actor-related primary data, or emerge much more informally from a project's own context.

Touchpoints can be considered at different levels of granularity: a smartphone or an app on that smartphone could both be suitable touchpoints. In the example given above, "Facebook group", "class debate", and "lecture notes" could then be touchpoints belonging to the "peer conversations" channel. Alternatively, a "Facebook group Website" and "Facebook group - App" distinction could be introduced if pragmatically valuable in respect to the goals at hand.

Touchpoints coincide with the individual elements produced in the practice of UX and discussed by interaction design and service design theory: a website, an interface, a kiosk, a staff member. As such, touchpoints are medium-specific. The content they handle on the other hand is often mediumaspecific, so touchpoints can belong to more than one channel. In the course design example given above, there may be a "lecture note" touchpoint that belongs to both the "peer conversation" channel, the one where student-produced information belongs, and a second channel where all teacher-produced content belongs. Such touchpoints allow actors to effectively move across channels.

Similarly, a "lecture note" could exist in digital format, in physical format, or both, effectively creating a connection between the two different spaces. In a cross-channel ecosystem, these thresholds between touchpoints are called "seams".

Seams have been identified elsewhere (Chalmers, Maccoll and Bell, 2003; Chalmers and Galani, 2004) in a number of different contexts, including mixed reality games. They represent either a discontinuity in the way an individual medium is experienced (Chalmers et al, 2004) or a connection between "the distributed components of a product/service" (Greenfield, 2007).

We maintain instead, following Resmini and Lacerda (2016), that seams play a most important role within the larger experience ecosystem. They allow the passage of content and information from touchpoint to touchpoint and across channels and enable actors to progress in their individual experience. Touchpoints that belong to more than one channel act as a seam between these. While touchpoint-totouchpoint movement is the primary concern of actors, enabling movement across channels through artfully crafted seams allows the designers to increase the level of integration of the ecosystem.

A number of taxi apps allow for example to pay rides by creating a seam between the taxi system and the traveller's credit card system. This results in no transactions, either in currency or electronic money, happening during or after the ride itself, effectively eliminating a touchpoint from the system and improving the UX. 
It is worth noting that while arguably a good experience needs to proceed unobstructed, this does not mean seams should be unperceivable always. There might be situations where a "bump" in the interaction is necessary, for example to warn the actor she is leaving a secure channel for an insecure one.

Cross-channel design is systemic in nature and pragmatic in scope: the identification of channels and the attribution of touchpoints to them is part of the design process and a highly contextual activity dependent on project data that cannot be performed in abstract.

While channels primarily deal in blended space terms with the ontology, the relationships between these elements contribute to the topology of the ecosystem, and are an integral part of the structure of the ecosystem itself.

\section{CASE STUDY: PUBLIC TRANSPORT COMMUTING}

In the course of an exploratory study conducted in 2015 on the public transport system in the city of Karlstad, Sweden (Lång and Schlegel, 2015), the heuristic approach introduced by Resmini and Rosati (2011) was used to assess the overall structure and appropriateness of the ecosystems centred around public transport commuting. This system was created by the interactions between the Karlstad public bus company, Karlstadsbuss, its multiple touchpoints, actors wanting to travel within Karlstad, and the city environment itself. "Is Karlstadsbuss providing a good experience for public commuting into the city centre" was the original formulation of the organizational stress point that served as a catalyst for the design process.

The main elements taken into initial consideration for the conceptualization of the cross-channel ecosystem itself in the course of the study were: (i) a number of individual actors, whose desired future state was framed through the activity of "commuting for work to the city centre"; (ii) the digital and physical and touchpoints offered by Karlstadsbuss, including the company's web presence, its mobile app, the ticketing system, its printed customer information, and its staff; (iii) the physical city environment, signage, distances, and layout.

Primary data was acquired through interviews, surveys, and direct observation on site. Actors were requested to describe what "commuting for work" meant for them. They were asked to consider what their general goal was (with results including for example "to travel comfortably", "to be on time", "to save money", "to protect the environment"), and to describe in detail how they navigated from home to work and back.

These descriptions allowed the team to assess what touchpoints were effectively used and which ones were not. They captured competing and ancillary products and services and their respective role in the ecosystem that Karlstadsbuss did not consider from their organizational perspective, such as thirdparty travel apps.

In all, the team interviewed 19 commuters using semi-structured interviews. Immediate evidence showed that actors were following personal, different paths even when their experiences shared all or most of the same touchpoints, as the sequence in which they were accessed varied from interviewee to interviewee.

Frequencies of use were calculated for the various touchpoints together with a rough topology detailing the seams between them. This allowed the team to highlight usage patterns and grade the touchpoints in order of actor-assigned importance. At the end of this process, these 19 individual paths where normalized and combined into three main paths, with a few rarely walked paths demoted to secondary concerns.

Figure 2 shows a blueprint of the main touchpoints in the ecosystem in respect to the key activities actors identify as part of the "commuting" experience. Rows represent the channels the touchpoints belong to. The blueprint details the most common normalized path actors travelled through the ecosystem.

Actors conceptualize their path as starting with the purchase of a bus card (the 'acquire ticket' activity sequence) at a kiosk, school, or at the Karlstadsbuss customer centre. This was true for $84 \%$ of the actors. Recharging this bus card happens on a loop here, when necessary, for $47 \%$ of the actors. Then actors typically obtain information on their journey through the travel planner $(73 \%)$. Next, they walk to the bus stop (95\%). Once their bus arrives, actors board and validate their trip at the bus card scanner (89\%).

\section{DISCUSSION}

While touchpoints were easily extracted from the interviews, from data acquired at Karlstadsbuss, and from observation on site, reaching an agreement on the channels was a laborious and delicate process. A number of compromises to accommodate the different points of view within the team and within the company were required.

\subsection{Cross-channel experiences}




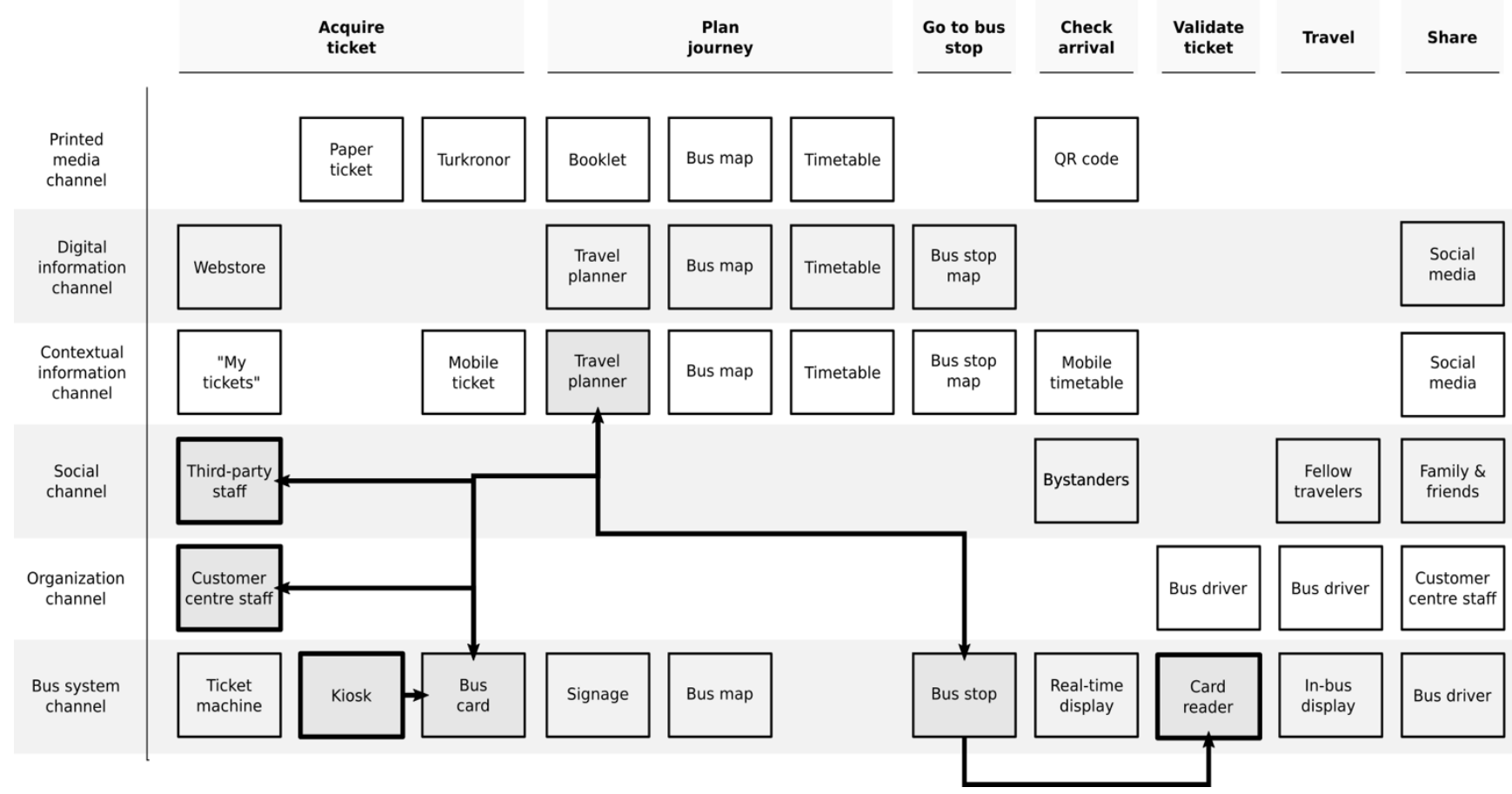

The study confirmed that all the actors interviewed were commuting for work using public transport by instantiating a cross-channel ecosystem in blended space. Actors exploited seams between non-

Figure 2: Blueprint of the touchpoints identified in the commuting experience. Rows show channels, columns activities

contiguous digital and physical spaces to create their own personal paths through the ecosystem.

Thirty-two individual touchpoints in six different channels were identified. The "printed media" channel, included paper tickets, booklets, QR codes,

Fuzzy channel boundaries were accepted as a necessary result of the sectioning criteria used to accommodate the organization's own view of their role in the ecosystem and facilitate understanding. For example, "printed media" was a compound channel resulting from splitting and reassembling parts of the more abstract "travel information", "company information", "environment information", and "traffic information" channels, to facilitate alignment with Karlstadsbuss's own conceptualization of their services.

The ecosystem was graded in terms of its capability to structure a comprehensive sense of place; to be conceptually consistent across the different mediums it was deployed through; to offer a degree of resilience and adaptability to individual idiosyncrasies, needs and wants; to reduce the cognitive load connected with choice; and to support

correlation as a way to expand an actor's potentiality for action (Resmini and Rosati, 2011). the bus timetables, the bus lines maps, and turkronor, a company-issued currency for tickets. The "bus system" channel, included physical touchpoints such as individual bus stops and digital touchpoints offering the possibility to verify the location of a given bus in real time. The most frequent path accesses six touchpoints across four different channels and contains one decision point that impacts the sequence in which activities are carried out. $78 \%$ of the actors accessed five or more of these six touchpoints, and all actors accessed at least three of them. $16 \%$ of the actors only accessed these three, in different sequences.

Having mapped the different paths allowed the team to create a rough representation of the shared commuting ecosystem and of its topology. These were then normalized into a series of synthetic views to which Resmini and Rosati's (2011) heuristics were applied to verify the degree of fitness of the current organizational strategy and efforts to the actual patterns of usage and role played by each element in the ecosystem.

\subsection{Blended Spaces}

In terms of the blended space approach, the key to good design lies in finding a structure for the generic space - in terms of its ontology, topology, volatility 
and agency - that will underlie the information architecture of the digital and the physical space and the correspondences between them.

The information architecture is distributed across the ecosystem, represented through the channels, accessed at touchpoints, together with information content coming from social and digital space, and effectively structured through the seams allowing movement within the ecosystem itself.

The ontology of the bus system explored in the study includes buses, bus stops, lines, routes, journeys, times and destinations. Clearly the physical space has bus stops and these have a topology in which bus stops have distance and directional relations with each other. On one line two bus stops may be adjacent whereas on another line they may be several stops apart. This topology is important as it affects, for example, the time it takes to get from one bus stop to another.

The volatility of the physical space is apparent in the movement of buses between bus stops. It is here where the relationships between buses and bus stops as described in the timetable may be disrupted by traffic delays.

We all know the frustration of waiting for a bus and not knowing where it is or when it will arrive. Agency in the physical space is provided by the bus drivers who can provide information about the space. Agency also includes understanding what people can do in the physical space and how they can affect it.

Designers might want to structure digital space to exploit the correspondences between it and physical space. So they might provide displays that show bus stops and their relationships on digital displays, on an app and at the bus stop itself (we observe here that the route maps at bus stops are part of an information space, but are not digital). By adding sensors to physical space, designers are able to track the volatile real-time movement of the buses, increasing the agency provided by the digital space and creating a seam across the two.

The blended space takes advantage of the correspondences between digital and physical spaces and now has its own properties that emerge from these relationships. In the blended space a commuter sitting on a bus can now have arrival times calculated. A person standing at a bus stop can have a display telling them how late the next bus will be. The agency of the blended space builds on the "commuting to work" function as the digital space enables emergent functions such as "get me home from here".

\section{CONCLUSIONS}

Cross-channel ecosystems are the result of actor usage and aggregation of any number of physical and digital touchpoints allowing their interactions with a pervasive layer of content. Touchpoints may be pertaining to one or more systems or competing services and are freely intertwined to pursue an implicit or explicit future state. An information architecture emerges that structures the topology of this ecosystem.

Cross-channel ecosystems instantiate co-produced blended spaces not bound by spatial contiguity but rather by semantic proximity and introduce one additional spatial characteristic not originally found in blended space theory, as blended spaces exist here as nested structures. More importantly, the whole ecosystem itself is a blended space: this implies that blends might happen between digital and physical spaces that are somehow separated and only connected semantically. These ecosystem-wide blended spaces support fluid, purposeful, seamful, stateful movement that can be modelled according to the principles of embodied cognition and conceptual blending.

Blending theory encourages designers to look at physical and digital spaces in a systemic manner. It brings into focus both physical and digital channels, how they can work together to provide new experiences, and how people can transition and move across channels to achieve their goals.

The formalization of cross-channel ecosystems supports and extends the four basic characteristics of blended spaces, ontology, topology, agency, and volatility, introduces a generative framing that takes into account co-production, and presents an opportunity for a more strategic approach to the design of user experiences.

Most importantly, the conceptualization of crosschannel ecosystems as blended spaces reaffirms the importance of a spatial, place-making approach to presence and navigation in user experience design, interaction design, and information architecture. The variance of context and the capability to act in blended space from a number of locations makes the creation of a sense of place an important component of design.

Designers need to help users find their way through the physical-digital mix and to cross boundaries. To do this they will look to principles of architecture and wayfinding (Arthur and Passini, 1992; Lynch, 1960). These principles have been used in information design (Jacobson, 2000), information architecture (Wurman, 1990), and in $\mathrm{HCl}$ (Benyon, Höök and Munro, 1997). Resmini and Rosati (2011) consider 
place-making a fundamental heuristic when designing cross-channel ecosystems and Benyon (2014) devotes a chapter to navigation. Although design to support navigation is good design more needs to be done to nudge users in the right direction and nudge them back on track when they lose their way.

In the case study we can see the importance of supporting user sense-making and navigation across the blended spaces. The bus card scanner must be clearly visible to make actors aware that they need to scan their card but also easy to operate and be able to communicate with the card and with remote systems. The travel planner, paper maps and interactive apps should present a consistent architecture across digital and physical spaces. The customer centre, bus stops, and kiosks should contribute to creating a sense of place together with the mobile app, the website, and the organization's digital presence. Users need to be aware of the opportunities offered by the different touchpoints that make up the ecosystem and of the role they play in the unfolding of their experience. The individual interactions are crucial to the success of the overall system, but of course they need to be designed together if the overall UX is to be regarded as successful. Furthermore, the UX will need to accommodate products and services that are outside of the company's control.

Also central to cross-channel ecosystems and blended spaces is information architecture. The information architecture constitutes the underlying, pervasive structure that gives designers the ontology that they can work with which in turn lets them look at the topology of the spaces and hence at important relationships such as separation, distance and direction. Information architecture provides the structure within which the UX unfolds.

User experience design needs to embrace the shift from the details of momentary interaction to the bigger picture suggested by the term 'architecture'. UX needs to fit its new interactions into legacy structures and accommodate them in enjoyable and aesthetic ways. UX needs to create places for interaction and to link places together through effective navigational design. Touchpoints need to attract people to engage in interactions through channels that fit their circumstances. Interactions may be interrupted and restarted on another channel, but it is the information architecture that makes this consistent and meaningful. This complex web of blended spaces and cross-channel ecosystems opens up the next era of user experience and interaction design.

\section{REFERENCES}

Arthur, P. and Passini, R. (1992) Wayfinding: People, Signs, and Architecture. Mcgraw-Hill.

Bannon, L. (2011) Reimagining $\mathrm{HCl}$ : toward a more human-centred perspective Interactions. 18(4). Pp. 50-57.

Beaudouin-Lafon's (2000) Instrumental Interaction: An Interaction Model for Designing Post-WIMP User Interfaces Proc CHI 2000.

Benford, S., Giannachi, G., Koleva, B., Rodden, T. (2009) From Interaction to Trajectories: Designing Coherent Journeys Through User Experiences. Proceedings of $\mathrm{CHI} 2009$.

Benyon, D. R. (2017) Designing User Experience; a guide to $\mathrm{HCl}, \mathrm{UX}$ and interaction design. Pearson

Benyon, D. R. (2014) Spaces of Interaction, Places for Experience. Morgan and Claypool.

Benyon, D. R. (2012) Presence in Blended Spaces Interacting with Computers. 24(4). Pp. 219-226.

Benyon, D. R., Höök, K., Munro, A. J. (1997) (eds.) Social Navigation of Information Space. Springer-

Benyon, D. R. and Mival, O. (2012) Designing Blended Spaces. Proceedings of Designing Collaborative Interactive Spaces. http://hci.unikonstanz.de/dcis/.

Benyon, D. R., Mival, O. and O'Keefe, B. (2013) Blended Spaces and Digital Tourism. Proceedings of CHI2013 workshop on Blended Interaction Spaces.

Bodker, S. (2015). Third-wave $\mathrm{HCl}, 10$ years later Participation and sharing. Interactions. 22(5). Pp. 24-31. http://dx.doi.org/10.1145/2804405.

Bodker, S., Klokmose, C.N. (2012) Dynamics in artifact ecologies. Proceedings of NordiCHI'12. Pp. 448-457.

Buxton, W. (2001). Less is More (More or Less), in P. Denning (ed.), The Invisible Future: The seamless integration of technology in everyday life. McGraw-Hill. Pp. 145-179.

Chalmers, M, Dieberger, A., Höök, K., and Rudström, A. (2004). Social Navigation and Seamful Design. Journal of the Japanese Cognitive Science Society

Chalmers, M. and Galani, A. 2004. Seamful interweaving: Heterogeneity in the theory and design of interactive systems. Proceedings of the ACM Conference on Designing Interactive Systems (DIS'04). Pp. 243-252. 
Chalmers, M., Maccoll, I., and Bell, M. 2003. Seamful design: Showing the seams in wearable computing. Proceedings of the IEEE Eurowearable Conference. Pp. 11-17.

Ciolfi, L. (2013) Space and place in digital technology research: A theoretical overview. In Price, S., Jewitt, C. and Brown, B. (eds.). The SAGE Handbook of Digital Technology Research. SAGE.

Ciolfi, L. (2004) Understanding Spaces as Places: Extending Interaction Design Paradigms. Cognition Technology and Work. Special Issue on "Presence: design and technology challenges for cooperative activities in virtual or remote environments". 6(1). Pp. 37-40. Springer.

Dalsgård, P. and Halskov, K. (2006) Real Life Experiences with Experience Design. Proceedings of Nordichi 2006.

Dalsgård P., Halskov K., Wiethoff A. (2016) Designing Media Architecture: Tools and Approaches for Addressing the Main Design Challenges. Proceedings of $\mathrm{CHI} 2016$.

Falck, H. W. and Halskov, K. (2013) Towards a Framework for Projection Installations. Proceedings of PerDis ' 13.

Fauconnier, G. and Turner, M. (2002) The way we think: Conceptual Blending and the Mind's Hidden Complexities. Basic Books.

Forlizzi, J. (2007). The product ecology: Understanding social product use and supporting design culture. International Journal of Design, 2(1), 11-20.

Forlizzi, J. and Battarbee, K. (2004) Understanding experience in interactive systems.

Gabriel-Petit, P. (nd) User experience design. http://www.uxmatters.com/glossary/.

Garrett, J. J. (2011) The Elements of User Experience. New Riders. 2nd ed.

Greenfield, A. (2007). On the ground running: Lessons from experience design. https://speedbird.wordpress.com/2007/06/22/onthe-ground-running-lessons-from-experiencedesign/.

Hassenzahl, M. (2008) User experience (UX): towards an experiential perspective on product quality. Proceedings of the 20th International Conference of the Association Francophone d'Interaction Homme-Machine. Pp. 11-15.

Hinton, A. (2015). Understanding Context. O'Reilly.
Hornecker, E. and Burr, J. (2016) Getting a grip on tangible interaction: a framework on physical space and social interaction. Proceedings of $\mathrm{CHI}$ 2016.

Hoshi, K. and J. Waterworth, J. (2009) Tangible Presence in Blended Reality Space. Proceedings of Presence 2009.

Hutchins, E. (2005) Material anchors for conceptual blends. Journal of pragmatics. 37(10). Pp. 15551577.

lacobacci, N. (2008). From crossmedia to transmedia: toughts on the future of entertainment.

http://www.lunchoverip.com/2008/05/fromcrossmedia.html.

Imaz M. and Benyon, D. (2007) Designing with Blends - Conceptual Foundations of HumanComputer interaction and Software Engineering. The MIT Press.

Jacobson, R. (2000) (ed.) Information Design. The MIT Press.

Jenkins, H. (2006). Convergence Culture. New York University Press.

Jenkins, H. (2011). Transmedia 202: Further Reflections.

http://henryjenkins.org/2011/08/defining_transmed ia_further_re.html.

Jetter, HC., Reiterer, H. and Geyer, F. (2014). Blended Interaction: understanding natural human-computer interaction in post-WIMP interactive spaces. Personal Ubiquitous Computing. Vol. 18. Issue 5. Pp. 1139-1158. http://10.1007/s00779-013-0725-4.

Jung, H. and Stolterman, E (2012) Digital Form and Materiality: Propositions for A New Approach to Interaction Design Research. Proceedings of Nordichi 2012.

Kirsh, D. (2000) A Few Thoughts on Cognitive Overload. Intellectica. 1(30). Pp. 19-51.

Kirsh, D. (2013) Embodied cognition and the magical future of interaction design. ACM Trans. Computer-Human Interactions. 20(1).

Lynch, K. (1960) The Image of the City. The MIT Press.

Lång, I. and Schlegel, A. (2015) Applying crosschannel user experience design theory to practice - A case study of a public transportation company in Sweden. Jönköping International Business School. 
Lucas, P, Ballay, J., and McManus, M. (2012). Trillions - Thriving in the Emerging Information Ecology. Wiley.

McCarthy, J. and Wright P. (2004). Technology as experience. The MIT Press.

Mitchell, W. J. (2004). Me++ The Cyborg Self and the Networked City. The MIT Press.

Norberg-Schulz, C. (1987). Existence, Space and Architecture. Praeger Publishers.

Norman, D. and Nielsen, J. (n.d.) The Definition of User Experience. Nielsen Norman Group. https://www.nngroup.com/articles/definition-userexperience/.

O'Keefe, B. and Benyon, D. R. (2016) Using the Blended Spaces Framework to design Heritage Stories with Schoolchildren. International Journal of Child-Computer Interaction.

Resmini, A. and Rosati, L. (2013) A Brief History of Information Architecture. Journal of Information Architecture. 3(2). Pp. 33-45.

Resmini, A. (2014) Reframing Information Architecture. Springer.

Resmini, A. (2013) The Architecture of Information. Études de communication, 41/2013. Pp. 31-56. Doi:10.4000/edc.5380

Resmini, A. and Lacerda, F. (2016) The Architecture of Cross-channel Ecosystems - From convergence to experience. Proceedings of the 8th International ACM Conference on the Management of Digital Ecosystems (MEDES'16).
Resmini, A. and Rosati, L. (2009) Information Architecture for Ubiquitous Ecologies. Proceedings of the International ACM Conference on Management of Emergent Digital Ecosystems (MEDES'09). Pp. 29:196-29.

Resmini, A. and Rosati, L. (2011) Pervasive Information Architecture: Designing Crosschannel User Experiences. Morgan Kauffman.

Rogers, Y. (2006) Moving on from Weiser's vision of calm computing: Engaging ubicomp experiences. Proceedings of Ubicomp 2006.

Rosenfeld, L. and Morville, P. (2006) Information Architecture for the World Wide Web. O'Reilly. 3rd ed.

Shedroff, N. (2010). An Evolving Glossary of Experience Design. https://web.archive.org/web/20150801125440/http ://www.nathan.com/ed/glossary/

Tuan, Y. (1977) Space and Place. University of Minnesota Press.

Vargo, S. L. and Lusch, R. F. (2004) Evolving to a New Dominant Logic for Marketing. Journal of Marketing. 68(1). Pp. 1-17.

Wang, H. H. (2013) A case study on design with conceptual blending. International Journal of Design Creativity. Volume 1. Pp. 109-122.

Winograd, T. (1996) Bringing Design to Software Addison-Wesley.

Wurman, R. S. (1990). Information Anxiety. Bantam.

Wurman, R. S. (1989) Hats. Design Quarterly. 